\title{
Imitation Control in Biped Robot by Using Controller to Operator
}

\author{
J. H. Lee, Y. F. Cui, S. G. Shon, and H. J. Byun
}

\begin{abstract}
This study describes a robot that can be remotely imitation controlled by an operator wearing a special suit with sensors to facilitate various movements. Remote controlled robot has quite different body structure and control strategy. Traditionally, robot is controlled by preset commands which are calculated from the requirements to keep balance. However, our robot control command is issued by operator instead of robot itself. Balancing imitation control algorithm is designed and implemented to walk without falling down. All the trajectories such as turn, stop, start, walk, etc, are determined by the operator. Finally, experiment result shows that this kind of new control method can be applied on various areas.
\end{abstract}

Index Terms-Balance control algorithm, biped robot, Imitation control.

\section{INTRODUCTION}

Around the world, interest in robots is increasing. Intelligent service robots have been a focus in the 21st century. Carry out personal tasks as pets and cleaners, and also carrying out work in areas such as security, medicine, guidance, disaster relief, and nuclear power. In addition, Robots do dangerous and difficult work in the place of people [1]. For example, bomb removal and the war on behalf of human beings are used for reconnaissance in the area.

Recently, study for humanoid robots is underway. A bipedal robot with excellent mobility has many potential applications. A humanoid robot has the outstanding ability to work with high mobility in the environment of human life. Incorporated into the everyday life of human is an important key [2]. It is necessary to build a learning system to do so in order to control a humanoid robot to imitate. Operator shall establish a system of artificial. So it can be useful in everyday life if you control a humanoid robot [3]. Thus, there is a need to be controlled humanoids robots by human.

Humanoid robot's intelligence is low. It will not be able to perform multiple tasks. So you need a way to control a humanoid robot. There are many ways to control a humanoid robot to Imitate. Imitation is a way to track the movements of the operator's control. There is a way by using ZMP, by using Sensor, by using a joystick and gesture. ZMP represents that

Manuscript received September 30, 2012; revised November 11 , 2012.This journal was supported by the GRRC SUWON 2012-B5 program of Gyeonggi province.

Yifenf Cui is with the College of Computer Science and Technology of Shandong University of Technology Linyi University of China (chiyifeng2008@gmail.com).

Junho Lee, Sugoog Shon and Heejung Byun are with the Department of Information and telecommunication Engineering, Suwon University, Korea (e-mail: junho_fly@naver.com; $\quad$ sshon@suwon.ac.kr; heejungbyun@suwon.ac.kr). a reaction to the force exerted on the soles of the feet become zero. By using ZMP, humanoid robot gait does not fall down [4]-[6]. Use sensors in order to control the robot to imitation control. The sensor used the acceleration sensor and a gyro sensor. Two sensors are attached to the waist and thighs. After collecting and analyzing the movement data of the operator, control the robot [7][8]. But how to use the sensors will need to collect a lot of data. Humanoid robots can be controlled with a joystick. Operators control the robot using a three-axis joystick. Rotation, pushing and pulling, etc. can be carried out. However, the robots have limited movement [9]. The image recognition can be controlled the robot. Provide an optimization framework to capture the behavior of human beings to create a movement of the upper body of a humanoid robot in. 2D movement and handwriting, and mimic the behavior of a simple task. However, this method is described as the movement of the arms and legs of the robot in cyberspace [10]-[12].

This paper presents a robot that can be remotely controlled by an operator wearing controller equipment. Reduce the data to be transmitted to the robot, a variety of movements and gait is possible through imitation control.

\section{CONSTRUCTION ABOUT IMITATION CONTROL SYSTEM}

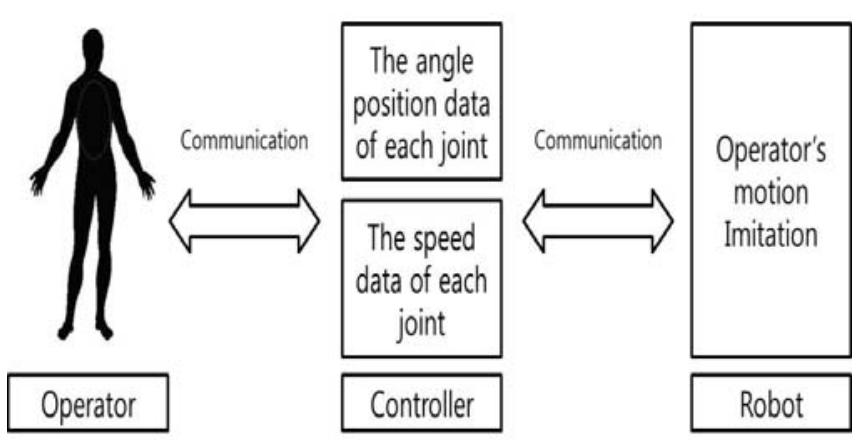

Fig. 1. Concept of imitation control system

We are proposed the imitation control system as shown in Fig. 2. The imitation control system consists of one computer, a biped robot and controller. The operator through controller controls the robot. The robot imitates the motion of the operator. After Operators recognize the surrounding environment and terrain, the motion and trajectory determine. And then, the operator has communication between the operator and the robot through controller. The data obtained by the operator will be sent to the robot actuators. When the robot starts to move, the robot's data is transferred to operator's controller. 


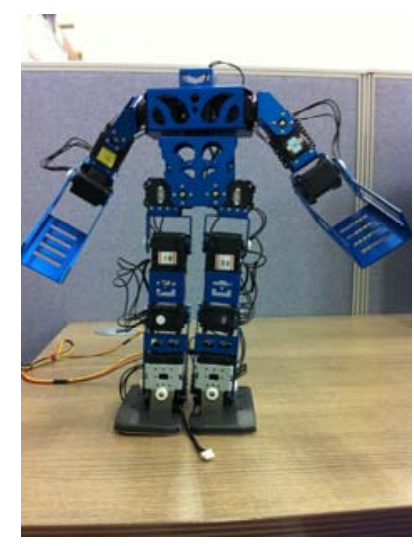

Fig. 2.The bipedal robot

The overall appearance of a humanoid robot represent in Fig. 2. Robot uses the whole body servo motor 17 . The upper body of the robot consists of 7 servo motors and the lower body of the 10 servo motors. The more servo motors the robot has, the more elaborately the robot copies movement of controller. However, too much motors increase weight of the robot hence this research used 17 motors to produce the robot. Servo motors is used ROBOTIS's Dynamixel AX-12A servo motor.

TABLE I: THE ROBOT SPECIFICATION

\begin{tabular}{cc}
\hline \hline Property & Value \\
\hline Height $(\mathrm{cm})$ & 35 \\
Weight $(\mathrm{g})$ & 909.5 (except of frame weight) \\
DOF & 17 \\
\hline \hline
\end{tabular}

As can be seen in Table I, the robot has an overall height of $35 \mathrm{~cm}$, weight 909.5g (except of frame) and a total of 17 degrees of freedom upper body 7 degrees of freedom, the lower body with 10 degrees of freedom.

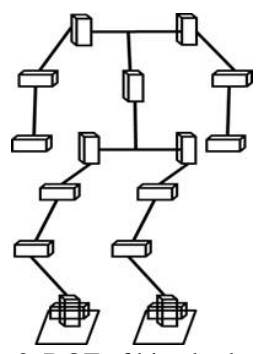

Fig. 3. DOF of biped robot

DOF represents the degrees of freedom. Fig. 3. shows 17 DOF of a biped robot. It has 17 DOF including vertical movement (pitch), rotation movement (yaw) and horizontal movement (roll) DOF. Lower body has 10 DOF (Degree of freedom). The 2 degrees of freedom in ankle, 1 degree of freedom in knee and 2 degrees of freedom in thigh are divided. Upper body has 7 DOF. 1 degree of freedom in the waist, 2 degrees of freedom in the shoulders and 1 degree of freedom in the cuffs are divided.

\section{BALANCE CONTROL OF BIPED ROBOT}

When human is walking, motion has way of two. Two legs to reach the floor and stand on one leg, his behavior do to move forward. But robot differs from the structure of the human. So, robot is necessary to move the center of gravity.

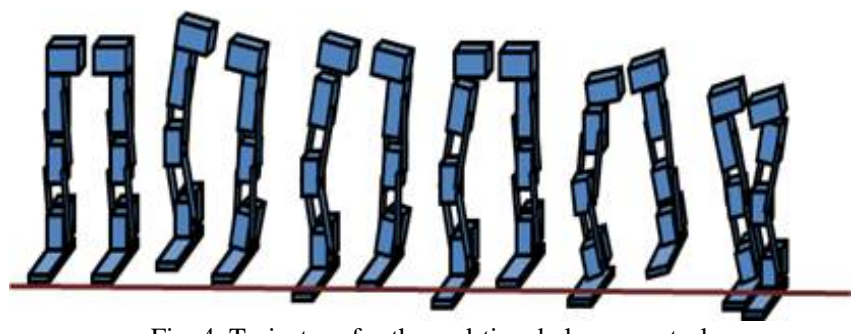

Fig. 4. Trajectory for the real-time balance control

Fig. 4 shows the robot's walking trajectory based on center of gravity and a test of the equipment. If you do not use this trajectory, the robot falls down. So, Biped walking requires movements as in Fig. 4. For one step forward, the left leg sup-port body when the right knee and thigh are bent and lifted up. Then, the center of gravity is drifted to the right side. With the center of gravity being put on the right foot, the left foot is controlled by left slope ankle so that robot can stand on one foot. It is one part of human walking. It repeats for forward movements. Some of the robot's joints are controlled to make trajectories by operator's command and some of the joints are used to keep the balance with COM algorithm. Decision to make movements such as walk, turn, stop and start is made by the operator. Once a person wearing controller equipment walks on two feet, robot receives the position values and performs the same biped walking.

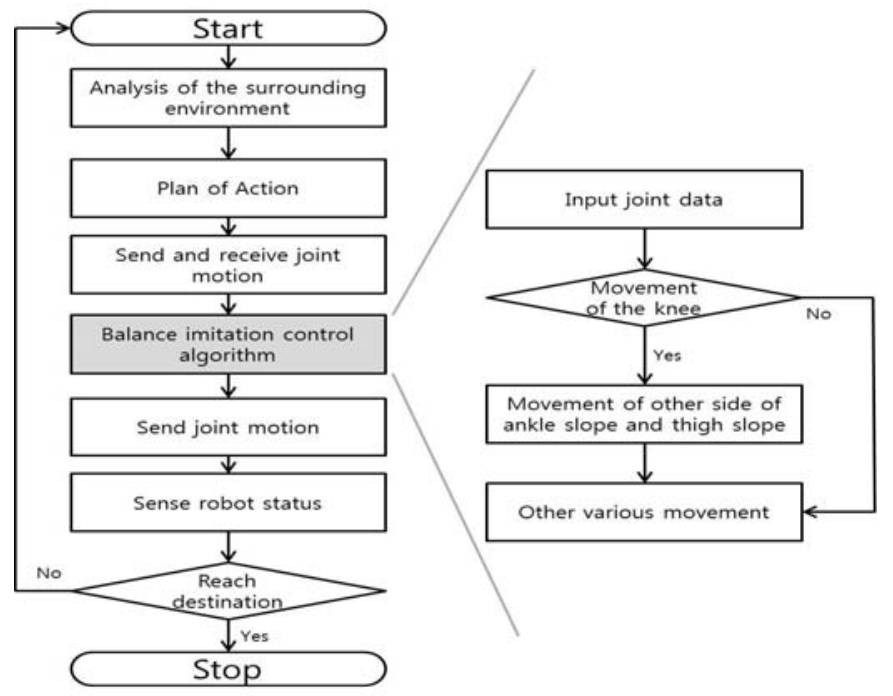

Fig. 5. Algorithm of balancing imitation control

Fig. 5 is the algorithm for the biped robot's walking. The operator recognizes the robot's surroundings, analyzes how to deal with each situation and plans how to act. Then, the operator's motion is transmitted through the controller. The controller transmits the operator's motion data to the robot's servo-motors, technically, via the balance imitation control algorithm. The balance imitation control algorithm works within the stable region. The operator's joint data entering the controller applies for walking. The robot needs to stand on one foot to walk. To walk, the robot needs to move the knee. Upon the knee moving, the tilt servo-motors on the robot's thighs and ankles move at the same time to keep its balance, which requires shifting the center of mass. Through this process, the robot keeps its balance, receives data and walks stably. it to stand on one foot, so as to take biped walking. 


\section{EXPERIMENT}

Experiment is supposed that operator's motion and robot's motion are performed on a flat floor.

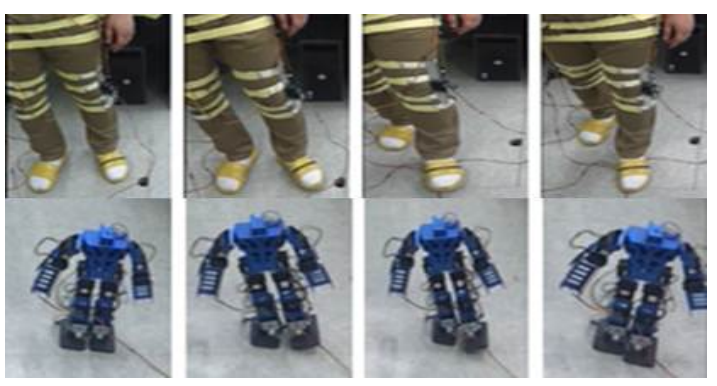

Fig. 6. the operator and the robot gait imitation control

Fig. 6 shows using the controller for operator to control the robot. Controller uses the sensor on the thigh and knee. Total is used 4 sensors. In the quiescent state if the operator lifts the knees, the ankles of the robot tilts. And the servo motor located in the thigh of the robot tilts to the side. In other words, if the operator to move the knee, robot's thigh servo motors and ankle servo motors move simultaneously. In this situation, if the opposite leg forward, the robot moves by imitation. And the operator moves the knee. After you do this, the robot's leg is landing on the ground. By repeating this method, the robot is able to walk. However, the robot has a slow gait.

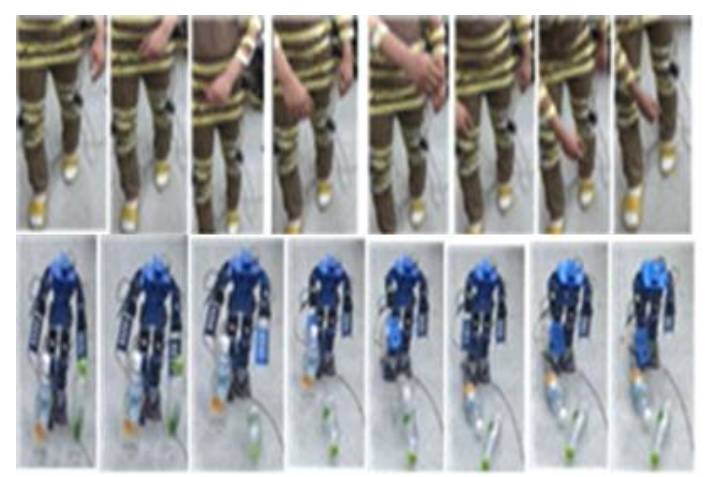

Fig. 7. the elimination of obstacles through imitation control

The operator identify the types of obstacles with own eyes. And decide whether any action. Fig. 7 shows that the operator controls the robot to remove the obstacles. The user can remotely control the robot's behavior to fit any situation. And remove two obstacles using the robot's left arm and right arm. Upper body controller is controlled by the six sensors. Arm-turn, arm-ups, etc. several movements are possible.

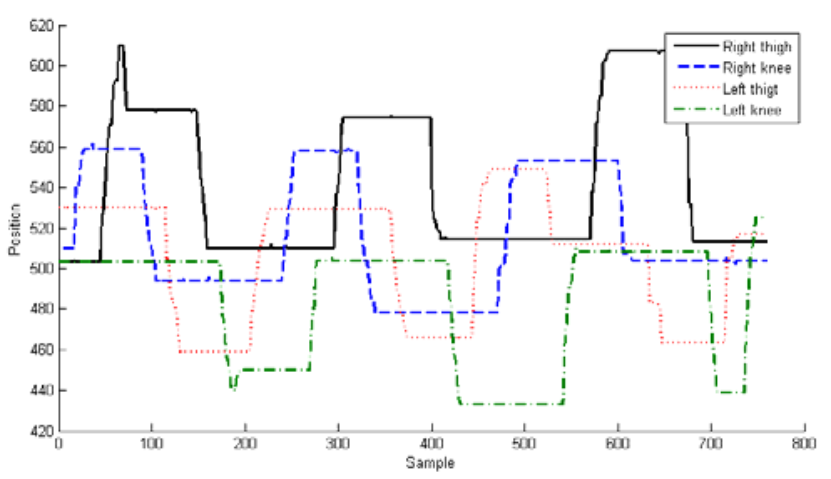

Fig. 8. 3 gait controller input data
Fig. 8 shows the input data of walking three steps using the controller to operator. The graph has four lines. Four graphs represent the right thigh, right knee, left thigh and left knee. The first step moves the knee. And next step moves the thigh forward, and opposite legs move. This method is performed repeatedly. In other words, the operator actually goes to the way of walking.

Fig. 9 is a graph showing the movement of the robot's thighs when the operator moved the right thigh using the controller. Operator imitates the robot control using controller. If you watch the graph, you can see that the relationship between input and output has a time delay. When moving the knee using a controller to operator, graph is showing the change of the robot's legs as shown in fig 10 . If operator moves knee, the slope of the robot thighs moves with the slope of the robot ankle. By moving the center of gravity, it is a process for stand on one foot. Fig.11 is a graph showing the movement of the right leg of the robot. Operator using the controller to control servo motors is used total four sensor. The controller has not the ankle, the thigh slope and the ankle slope sensor. We control the four servo motors of robot using a controller which has two sensors.

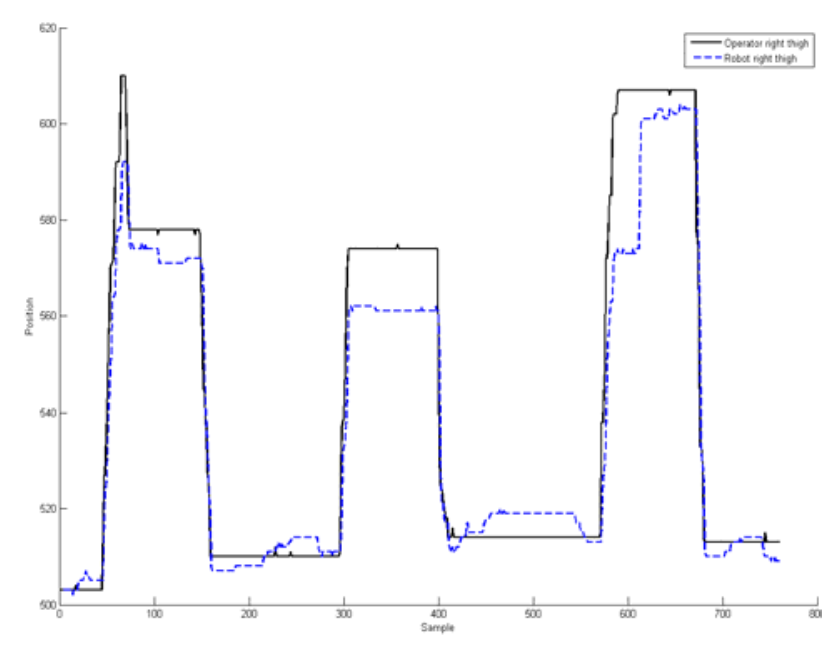

Fig. 9. Comparison of thigh data in controller and robot

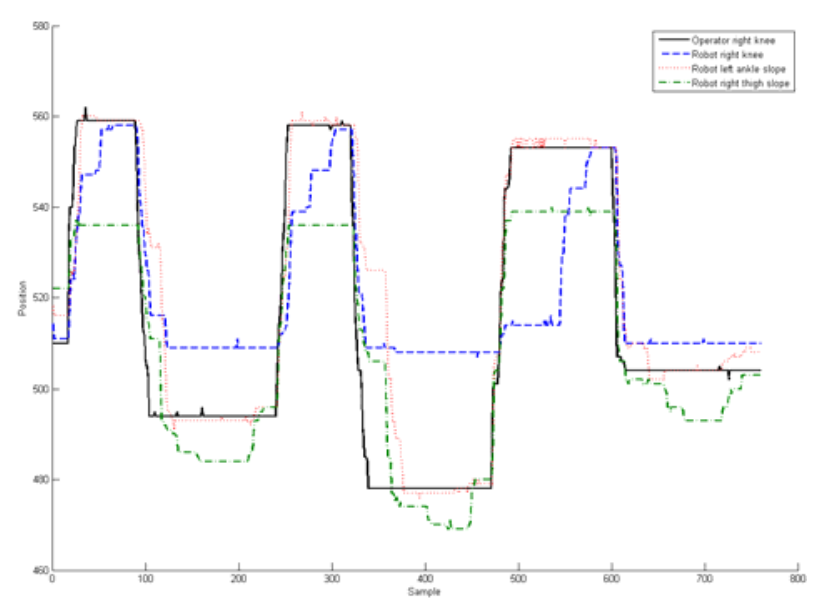

Fig. 10.Comparison of knee data and ankle slope 


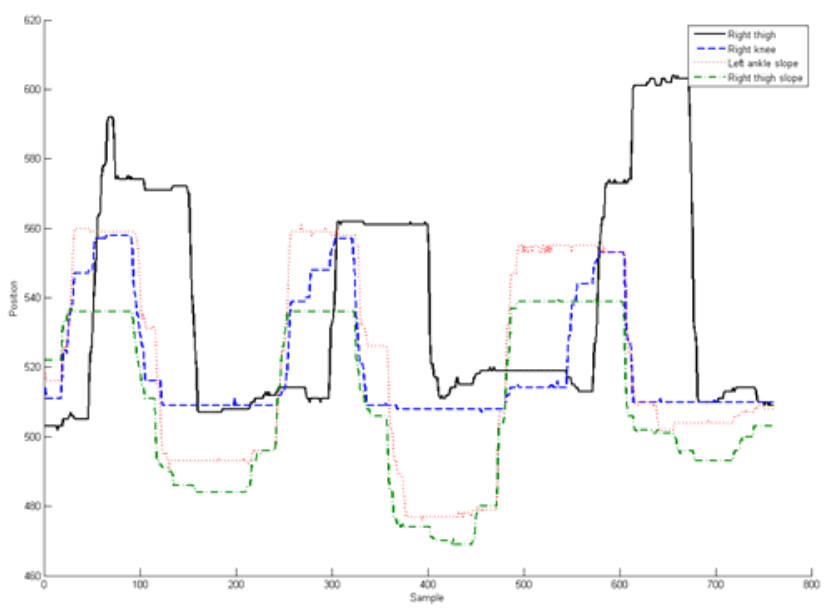

Fig. 11. Thigh, knee, ankle slope and thigh slope data of robot

\section{CONCLUSION}

This research shows that the bipedal robot can walk without falling down even though it is controlled by human remotely. Input commands to determine its trajectories are issued by the encoders attached to operator's joints as the operator moves and transferred to the robot through the communication path. It is quite different from that the traditional robot generates trajectories calculated from its kinematics for every joint. As for the control strategy, some of the robot's joints are controlled to make trajectories by operator's command and some of the joints are used to keep the balance with imitation control algorithm. Decision to make movements such as walk, turn, stop and start is made by the operator. Experiments show that the robots can move freely as the operator want to. However, there were difficulties related to balancing its movement, due to vibrations, and the robot moved slowly. Further research will focus on improving the robot's walking speed and improved ramps up to develop an algorithm for complementary equipment to avoid losing balance.

\section{REFERENCES}

[1] Sul Cho, Rai Wung Park, "A study on Control Design and Production in Walking Robot with 2 Legs,” Daebul University Press, Korea, 2009, pp.363-378.

[2] Jong Hyeon Park, Hoam Chung, "ZMP Compensation and Impedance Control for Improving Walking Stability of Biped Robot," KSME, 2000, vol. 24, no. 4, pp.1007-1015.

[3] Cynthia Breazeal, Brian Scassellati, "Challenges in Building Robots That Imitate People,” MIT Artificial Intelligence Laboratory, Cambridge.

[4] Yong Soo Jang, "A Study on the Performance Improvement of Straight Walking of a Biped Robot by Using Vision and ZMP Feedback," Kyung Hee University press, Korea, 2009.
[5] Miomir Vukobratovic, "Zero-moment point - Thirty five years of ITS life," International Journal of Humanoid Robotics, vol.1, no.1 (2004), pp.157-173.

[6] Deok-Hyeon Ko, "Study on Energy Efficient and Robust Dynamic Walking Algorithm for a Biped Robot Using ZMP based Postural Control and Adaptation of Gait Trajectory,” Kyung Hee University, 2011, pp.13-24

[7] Yoshio Inoue, Kyoko Shibata, "Imitaion Control for Biped Robot Using Wearable Motion Sensor,” Kochi University of Technology, Japan.

[8] Weijun Tao, Tao Liu, Rencheng Zheng and Hutian Feng, Gait Analysis Using Wearable Sensors, pp. 2255-2283.

[9] Sechoon Ha, Yunfei Bai and C.Karen Liu, "Human Motion Reconstruction from Force Sensors,” The Eurographics Association, 2011.

[10] Wael Suleiman, Eiichi Yoshida, Fumio Kanehiro, Jean-Paul Laumond and Andre Monin, "On Human Motion Imitation by Humanoid Robot," LAAS-CNRS, AISY, France, Japan.

[11] C.A.Acosta-Calderon, H.Hu, "Robot imitation: Body schema and body percept,” ABBI 2005, vol.2, no.3-4, pp. 131-148.

[12] Mike stilman, Koichi Nishiwaki and Satoshi Kagami, "Humanoid Teleoperation for Whole Body Manipulation,” ICRA’ 08, 2008.

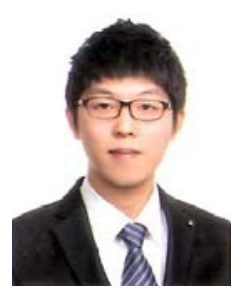

Junho Lee received the B.S. degree (2011) in Department of Information and Telecommunication from the University of Suwon. His research interests include computer and embedded system, Biped walking robot control system. He is currently studying to receive M.S. degree in Department of Information Telecommunication from the University of Suwon.

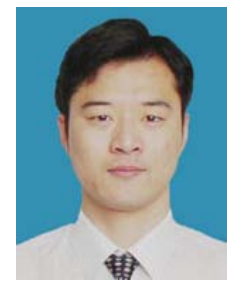

Yifeng Cui received the B.S. (2003) and M.S. (2007) degree in the College of Computer Science and Technology of Shandong University of Technology, he is a lecturer in the Linyi University of China. Now, he is studying for a doctor's degree in the University of Suwon in Korea. His research interests include computer measurement and control, network engineering and robot control system.

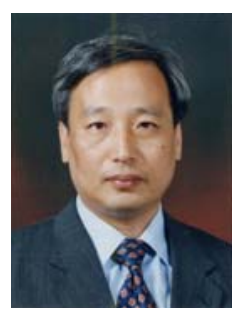

Sugoog Shon received his B.S. (1982) degree in Electrical Engineering from Seoul National University, his M.S. (1984) degree in Electrical Engineering from Seoul National University, and his Ph.D. (1996) degree in Electrical and Computer Engineering from the University of Texas at Austin. $\mathrm{He}$ is a professor in the Department of Information and Telecommunication at the University of Suwon in Korea. His research interests include computer and embedded system, network protocol, network simulation, and network programming.

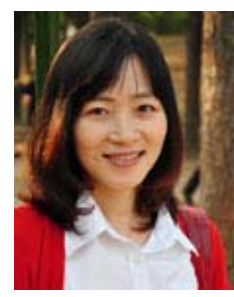

Heejung Byun received the Ph.D. degrees from Korea Advanced Institute of Science and Technology (KAIST), Daejeon, Korea, in 2005. She was a senior engineer in Samsung Electronics, Ltd. From 2007 to 2010. She is currently an assistant professor with the Department of Information \& Communications, Suwon University, Kyunggi-do, Korea. Her research interests include network modeling, controller design, and performance analysis. 